\title{
Fluorescence Enhancement of Immunoassay Using Multilayered Glass Substrates Modified with Plasma-Polymerized Films
}

\author{
Kazuyoshi Yano*, Kazuyuki Yamano, Akira Iwasaki, \\ Takuo Akimoto, Hirotaka Miyachi ${ }^{1}$ and Atsunori Hiratsuka ${ }^{2}$ \\ School of Bioscience and Biotechnology, Tokyo University of Technology, \\ 1401-1 Katakura, Hachioji, Tokyo 192-0982, Japan \\ 'Department of Clinical Engineering, School of Health Sciences, Tokyo University of Technology, \\ 5-23-22 Nishikamata, Ota-ku, Tokyo 144-8535, Japan \\ ${ }^{2}$ Smart Sensing Group, Nanosystem Research Institute, \\ National Institute of Advanced Industrial Science and Technology (AIST), \\ Central 4, 1-1-1 Higashi, Tsukuba, Ibaraki 305-8562, Japan
}

(Received February 23, 2015; accepted May 13, 2015)

Key words: multilayered substrate, fluorescence enhancement, plasma polymerization

The plasma polymerization technique, which enables deposition of thin and pinhole free films, was employed to fabricate multilayered substrates for fluorescencebased sensitive immunoassay. A glass slide was modified with a 200-nm-thick Ag layer as a metal mirror and further coated with plasma-polymerized films (PPFs) up to 120 $\mathrm{nm}$ thick as the optical interference layers. Cy3-labeled antibody was spotted on the resulting multilayered substrate and the fluorescence image was observed. The Cy3 fluorescence was highly enhanced on the multilayered substrate compared with that observed on the area without PPFs. The maximum fluorescence intensity was obtained when PPF thickness was $63 \mathrm{~nm}$, showing a 30-fold enhancement of fluorescence compared with that obtained from the unmodified substrate. The multilayered substrates with PPF on which multiple proteins were immobilized were further utilized to perform immunoassays, resulting in specific and highly enhanced fluorescence detection.

\section{Introduction}

Fluorescence-based biosensing is one of the most popular techniques employed in the fields of molecular biology, the life sciences, and clinical testing. Generally, detection is achieved by utilizing fluorophores to label the biomolecules and by measuring the fluorescence intensity upon binding events between the labeled molecules and their targets. Therefore, the enhancement of the fluorescence intensity is highly important to improve sensitivity and to lower the detection limits. A number of methodologies to

*Corresponding author: e-mail: yano@stf.teu.ac.jp 
enhance fluorescence have been developed, such as an immunoassay using a substrate modified with high-aspect-ratio nanopillars ${ }^{(1)}$ and an affinity-based assay employing an environmentally sensitive dye.(2) Enhancement can be also achieved by an optical phenomenon called metal-enhanced fluorescence (MEF), which is induced by localized surface plasmon resonance (LSPR) on metal nanoparticles. ${ }^{(3,4)}$ Due to its great potential, MEF has recently received considerable attention and been exploited in highly sensitive bioanalytical applications, such as DNA-based assays, ${ }^{(5-7)}$ immunoassays,${ }^{(7,8)}$ and other protein assays. ${ }^{(9,10)}$ However, MEF-based sensing systems generally require fabrication of three-dimensional nanostructures involving precise control of size, shape, and density of surface structures.

Another simple technique to enhance fluorescence is based on the optical interference phenomenon on glass slides modified with a plane metal mirror coated with an optical interference layer. The first multilayered substrate reported was fabricated with approximately 100-nm-thick dielectric LiF as an optical interference layer on the surface of a Ag mirror; this achieved a maximum 400-fold enhancement of fluorescence for Rhodamine B. ${ }^{(11,12)}$ The enhancement effect is explained by the double interference of both the excitation light and fluorescence in the optical interference layer, which may account for the fact that the fluorescence enhancement is periodically dependent on the thickness of the optical interference layer. Other multilayered substrates have been also reported employing $\mathrm{Al}_{2} \mathrm{O}_{3}{ }^{(13-17)}$ and $\mathrm{SiO}_{2}{ }^{(9,18)}$ as the materials of the optical interference layers instead of $\mathrm{LiF}$; these have been applied to affinity assays, ${ }^{(9,17)}$ mutagen detection, ${ }^{(16)}$ and cell imaging. ${ }^{(13)}$

In this study, we propose plasma-polymerized films (PPFs) as new alternatives to $\mathrm{Al}_{2} \mathrm{O}_{3}$ or $\mathrm{SiO}_{2}$ as optical interference materials for multilayered substrates. PPFs can be formed in a glow discharge or plasma in a vapor phase. They are free of pin-holes, adhere strongly to a wide range of materials, and are chemically and mechanically stable. ${ }^{(19)}$ A number of bioanalytical applications of PPFs for the modifications of sensor areas have been reported, ${ }^{(20,21)}$ such as immunoassays, ${ }^{(22,23)}$ single nucleotide polymorphisms (SNPs) typings, ${ }^{(24,25)}$ amperometric biosensors, ${ }^{(26-28)}$ capillary electrophoresis, ${ }^{(29)}$ and protein patterning. ${ }^{(30)}$ One of the remarkable features of PPFs is that protein molecules can be immobilized in PPFs without any loss of activity from direct exposure to plasma. We have demonstrated that streptavidin ${ }^{(24,25,31)}$ - and antibody ${ }^{(23)}$-embedded PPFs deposited on glass slides using hexamethyldisiloxane (HMDS) as a monomer can function as receptors for biotinylated DNA in DNA arrays and for antigens in protein chips, respectively.

Here we extend the excellent potential of PPFs to the application of fluorescence enhancement using multilayered substrates. PPFs are extremely thin $(<1 \mu \mathrm{m})$, and their thickness can be easily tunable simply by changing deposition time. Furthermore, thin HMDS PPF is transparent. Therefore, HMDS PPFs would be an excellent alternative to serve as the functional optical interference layer for fluorescence enhancement. Our approach for enhanced immunoassay is shown in Fig. 1. A Ag layer and subsequent PPF using HMDS as a monomer are deposited on a glass slide as a metal mirror and an optical interference layer, respectively, to obtain a multilayered substrate. After immobilization of antigen proteins on the surface of the substrate, Cy3-labeled antibody is added to allow specific interactions with the proteins and enhancement of fluorescence intensity. 


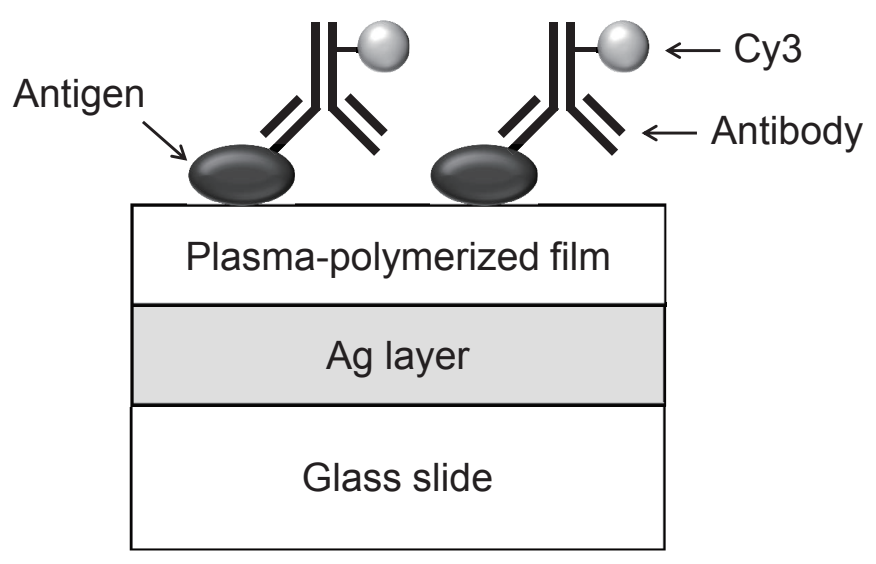

Fig. 1. Schematic diagram of a sensitive immunoassay using a multilayered substrate. A glass slide was modified with a Ag layer as the metal mirror and further coated with plasma-polymerized films as the optical interference layers. Immunoassays were performed on the surface of the substrate using a Cy3-labeled antibody to obtain enhanced fluorescence signals.

\section{Materials and Methods}

\subsection{Reagents}

HMDS was purchased from Shin-Etsu Chemical Co., Ltd. (Japan). Goat-derived Cy3-labeled anti-mouse IgG antibody was from GE Healthcare Biosciences (USA). Mouse IgG and rabbit IgG were purchased from Sigma-Aldrich (USA). Mouse-derived anti-human C-reactive protein (CRP) monoclonal antibody and rabbit-derived antihuman CRP polyclonal antibody were purchased from Oriental Yeast Co., Ltd. (Japan). Other reagents were of analytical grade from Wako Pure Chemical Industries (Japan).

\subsection{Deposition of metal layers on glass substrate}

A glass slide $\left(76 \times 26 \mathrm{~mm}^{2}\right.$, Matsunami Glass Ind. Ltd., Japan $)$ was cleaned by sonication in ethanol for $20 \mathrm{~min}$, washed in ultrapure water, and dried. After a 15-nmthick Cr layer as an adhesive was deposited onto the cleaned slide, a 200-nm-thick Ag layer as the metal mirror was formed. Both layers were fabricated using a sputtering apparatus (CFS-4ES, Shibaura Mechatronics Corp., Japan), and their thickness were measured with a surface profile meter (Dektak8, Veeco, USA).

\subsection{Apparatus for plasma-polymer deposition}

PPFs were prepared using a plasma deposition system (Model BP-1, Samco Inc., Japan), the schematic diagram of which is shown in Fig. 2. Two external electrodes were set along the vacuum chamber horizontally, $15 \mathrm{~cm}$ above the sample stage. A radio frequency (RF) generator (Model RFG-300, Samco), coupled to a matching box 


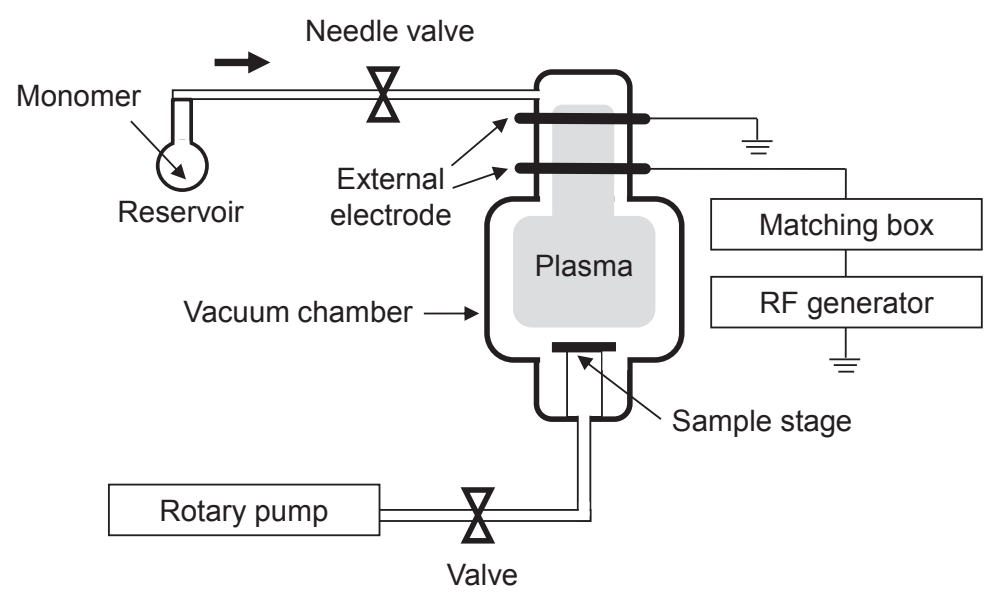

Fig. 2. Apparatus for plasma polymerization.

to minimize reflected power, was also employed. The working frequency of the power supply was $13.56 \mathrm{MHz}$.

\subsection{Preparations of PPFs}

First, the relationship between the deposition time for plasma polymerization and the film thickness was analyzed. A glass slide was placed on the sample stage of the plasma deposition apparatus. The chamber was evacuated, and a carrier gas of HMDS as a monomer was introduced from a reservoir at a constant flow rate of $20 \mathrm{ccm}$ maintained by a needle valve. The background pressure was approximately 0.4 Torr. Then plasma was discharged at an RF power of $100 \mathrm{~W}$ to polymerize and deposit the PPF on the substrate. A number of PPFs of different thicknesses at different areas on a single substrate were prepared by varying the deposition time. The thickness of each film was measured using an ellipsometer (ESM-1T, ULVAC, Japan), and a calibration curve of thickness against deposition time was prepared.

A multilayered substrate for fluorescence enhancement was similarly prepared on a glass slide coated with a 200-nm-thick Ag layer. The deposition time was set according to the calibration curve, and the deposition was analyzed every time by measuring the thickness of PPFs formed on small reference silicon chips placed on the same sample stage.

\subsection{Fluorescence measurement of Cy3-labeled antibody on the multilayered substrate}

Cy3-labeled anti-mouse IgG antibody was dissolved and diluted in phosphatebuffered saline (PBS, pH 7.4) to obtain $1.6 \mu \mathrm{g} / \mathrm{ml}$ solution. Then $1 \mu \mathrm{l}$ of the antibody solution was spotted on the surface of the multilayered substrate manually using a 
micropipetter. Three spots on each PPF with different thickness were made using the same aliquot and dried. Two-dimensional fluorescence images were obtained using a scanner (Pharos FX, Bio-Rad, USA), and the fluorescence intensities were analyzed using QuantOne Software (Bio-Rad).

\subsection{Immunoassay on the multilayered substrate}

Mouse IgG and rabbit IgG were dissolved in PBS to obtain $1 \mathrm{mg} / \mathrm{ml}$ solutions and were further diluted to desired concentrations. IgG samples of $2 \mu \mathrm{l}$ were spotted on four substrates with different modifications: unmodified, modified with a 200-nm-thick Ag layer only, modified with a 65-nm-thick HMDS PPF only, and modified with a 65-nmthick HMDS PPF on a 200-nm-thick Ag layer. After drying, the substrates were blocked by $400 \mu \mathrm{l}$ of blocking solutions [20 mM HEPES (pH 7.4), $137 \mathrm{mM} \mathrm{NaCl}, 30 \mathrm{mM} \mathrm{KCl}$, $5 \mathrm{mM} \mathrm{MgCl}, 0.1 \%$ Tween 20, and $1 \mathrm{mg} / \mathrm{ml}$ human serum albumin (HSA)] for $15 \mathrm{~min}$ and washed with PBS twice, each time for $2 \mathrm{~min}$. Cy3-labeled anti-mouse IgG antibody (400 $\mu \mathrm{l} \mathrm{of} 10 \mu \mathrm{g} / \mathrm{ml}$ solution) was added on the surface of the substrates, incubated for 15 min, washed with PBS twice, each time for 2 min, and dried. All immunoassay procedures were performed at room temperature. A two-dimensional fluorescence image was recorded by the scanner, and the fluorescence intensity of each spot was calculated by subtracting the background signals of each substrate.

Immunoassay targeting antibodies for CRP on the multilayered substrate was also performed using those derived from different animal sources. Mouse-derived anti-human CRP monoclonal antibody and rabbit-derived anti-human CRP polyclonal antibody were dissolved and diluted in PBS to obtain 10 and $1 \mu \mathrm{g} / \mathrm{ml}$ solutions each. Each sample $(1 \mu \mathrm{l})$ was spotted on an unmodified substrate and on one modified with a 61-nm-thick HMDS PPF on a 200-nm-thick Ag layer. Samples of mouse IgG and rabbit IgG solutions of the same concentrations were also spotted to increase the number of targets for preparation of protein arrays and to evaluate the selectivity.

\section{Results and Discussion}

\subsection{Determination of optimal thickness of PPFs for fluorescence enhancement}

First, the calibration curve for PPF deposition was obtained by changing the operating time. As illustrated in Fig. 3, the curve showed good correlation between deposition time and thickness of PPF. The deposition rate was approximately $0.33 \mathrm{~nm} / \mathrm{s}$. The deposited HMDS PPF had hydrophobic characteristics as reported previously. ${ }^{(32)}$

The principle of fluorescence enhancement applied in this study is based on the optical interference phenomenon at the dielectric layer on a substrate, which implies that the thickness of the layer is highly important. Therefore, the relationship between the thickness of the PPF on the multilayered substrate and the fluorescence intensity of the fluorophores spotted was evaluated to determine the optimal thickness of the PPF. A glass slide modified with a 200 -nm-thick Ag layer was further coated with various thicknesses of PPF at separate areas. Cy3-labeled anti-mouse IgG antibody ( $1 \mu \mathrm{l}$ of 1.6 $\mu \mathrm{g} / \mathrm{ml}$ solution) was spotted, allowed to physically absorb, and dried. Subsequently a two-dimensional fluorescence image was collected by the scanner without any wash 


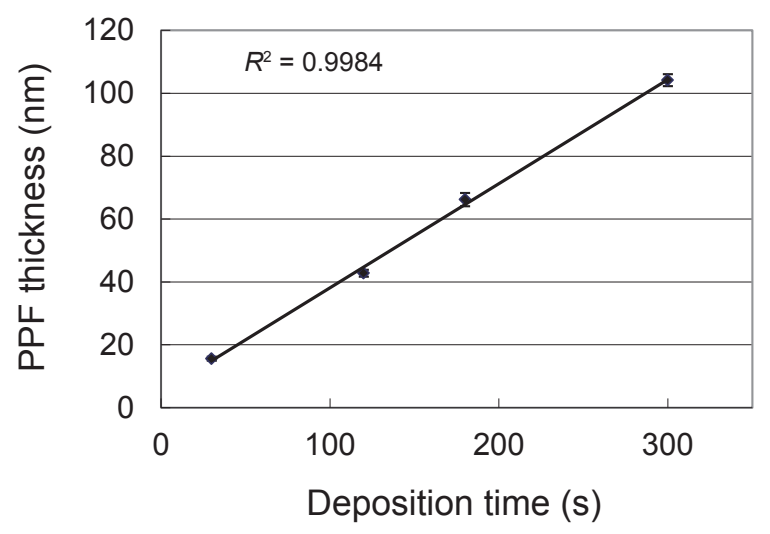

Fig. 3. Calibration curve for PPF thickness as a function of deposition time. Error bars indicate the standard deviation of 7 measurements.

treatment. The fluorescence image showed that the fluorescence of the Cy3 moiety of the labeled antibody was highly enhanced on the multilayered substrate compared with that observed on the area without PPF [Fig. 4 (a)]. Interestingly, the degree of enhancement was dependent on the PPF thickness; the maximum fluorescence intensity was obtained when the PPF thickness was $63 \mathrm{~nm}$ [Fig. 4 (b)], indicating a 30-fold enhancement of fluorescence compared with that obtained from the unmodified substrate.

A multilayered substrate looks like a mirror because of the presence of the $\mathrm{Ag}$ layer coated with transparent HMDS PPF. Therefore, it might be possible that the enhancement effect was simply attributed to the reflection at the surface of the Ag layer. However, the fluorescence observed on 0-nm-thick PPF, that is, on a Ag layer only, was very small, indicating that the presence of PPF of an appropriate thickness was essential for the 30-fold enhancement of fluorescence intensity. Aluminum might be used as an alternative for a metal mirror because of its high reflectivity. ${ }^{(18)}$

As for the $\mathrm{Al}_{2} \mathrm{O}_{3}$ layer, the thickness that provides peaks for fluorescence enhancement has been analyzed for various fluorophores. ${ }^{(15)}$ In the range from 0 to 100 -nm-thick $\mathrm{Al}_{2} \mathrm{O}_{3}$, the maximum intensity for $\mathrm{Cy} 3$ was obtained with a layer $91 \mathrm{~nm}$ thick, which was almost same as the calculated value. In this case, however, only the $\mathrm{Cy} 3$ molecule dissolved in ultrapure water was utilized to measure the fluorescence intensity and to simply evaluate the enhancement effect, whereas Cy3-labeled antibody solution in PBS was spotted on the HMDS PPF in this study. Therefore, the presence of a bulkier protein moiety in the labeled molecule, as well as the refractive index of HMDS PPF, which is possibly different from that of an $\mathrm{Al}_{2} \mathrm{O}_{3}$ layer, might affect the optimal layer thickness.

\subsection{Effect of multilayered substrate on the immunoassay}

As the multilayered substrate modified with appropriate thickness of PPF was shown to be effective for fluorescence enhancement, it was then utilized in the immunoassay format to evaluate whether enhanced detection of target protein could be achieved. Four 


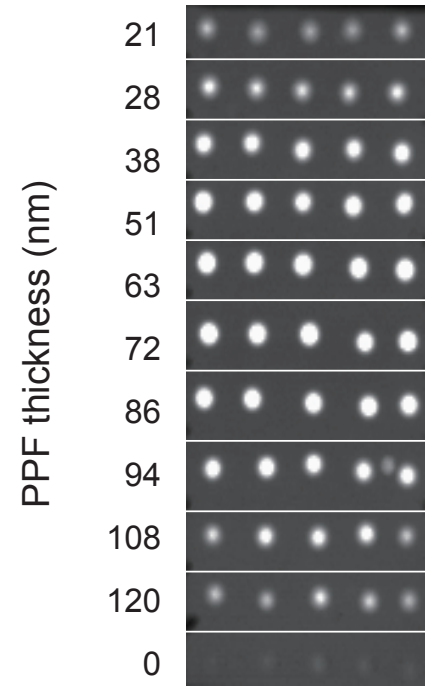

(a)

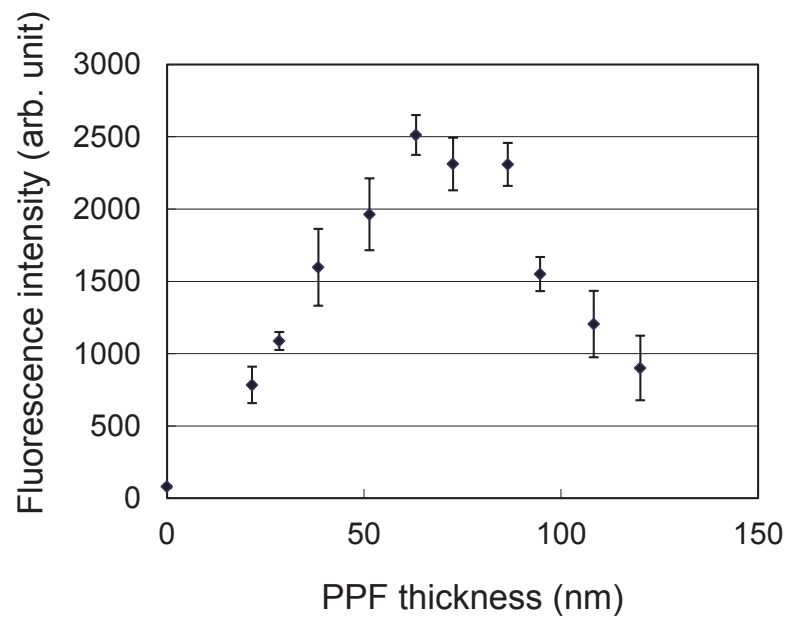

(b)

Fig. 4. Enhancement of fluorescence intensity from Cy3-labeled antibody on the multilayered substrate. (a) Two-dimensional fluorescence image of the multilayered substrate with various PPF thicknesses. Before plasma polymerization, the glass slide was precoated with a 200 -nm-thick Ag layer. Cy3-labeled anti-mouse IgG antibody ( $1 \mu \mathrm{l}$ of $1.6 \mu \mathrm{g} / \mathrm{ml}$ solution) was spotted and dried. (b) Relationship between PPF thickness and fluorescence intensity. Error bars indicate the standard deviation of 5 measurements.

substrates were prepared: unmodified, modified with a Ag layer only, modified with a 65-nm-thick HMDS PPF only, and modified with a 65-nm-thick HMDS PPF on a Ag layer. Immunoassays were performed on those substrates under the same conditions using physically-immobilized mouse $\operatorname{IgG}$ and rabbit $\operatorname{IgG}$ as an antigen and a negative control, respectively, and Cy3-labeled anti-mouse IgG antibody as a probe.

Two-dimensional fluorescence images showed that the fluorescence signal from mouse IgG was highly enhanced on the multilayered substrate, whereas the fluorescence intensities from 3 other control substrates were quite low or undetectable [Fig. 5 (a)]. No signal was observed from rabbit $\operatorname{IgG}$ even from the multilayered substrates, indicating specific enhancement only by the multilayered structure. The background fluorescence observed only with the multilayered substrate might be due to the reflection of the excitation light for $\mathrm{Cy} 3$ and further enhancement by the multilayered structure. Insufficient washing during the immunoassay procedures would be also a factor. In any case, however, much greater fluorescence intensity with the multilayered substrate in comparison to that with others is obvious.

The enhancement factors of the fluorescence were calculated by dividing the intensities obtained with the modified substrates by those obtained with the unmodified glass substrate and are summarized in Fig. 5 (b). It was shown that the fluorescence 


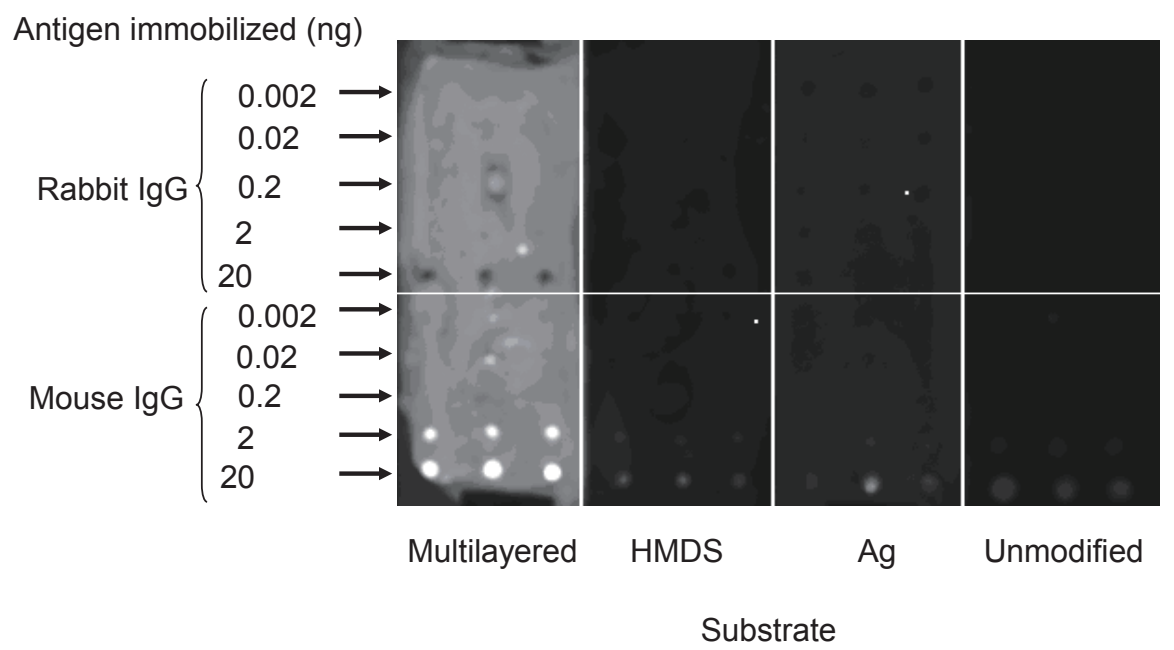

(a)

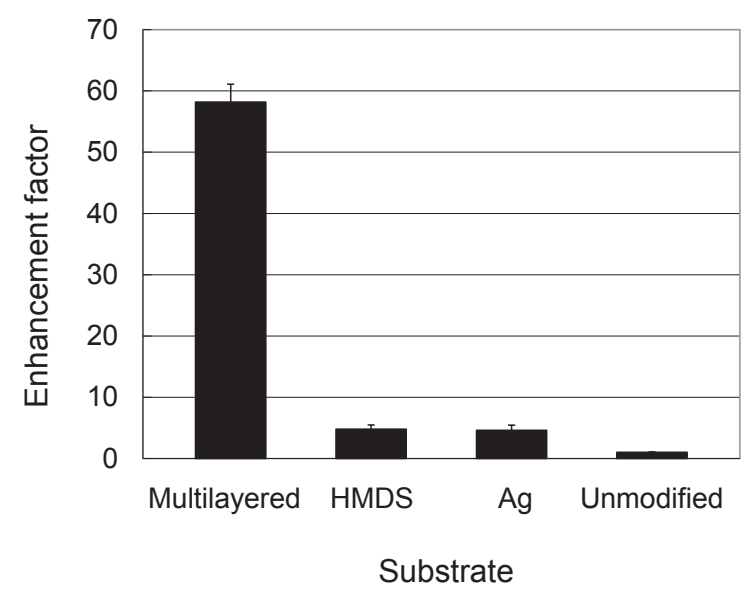

(b)

Fig. 5. Effect of the multilayered structure on the immunoassay. Antigen-antibody interaction was evaluated using Cy3-labeled anti-mouse IgG antibody on the multilayered or unmodified substrate and on the glass slide modified ony with 65-nm-thick HMDS PPF or 200-nm-thick Ag. (a) Two-dimensional fluorescence images of 4 substrates scanned after immunoreaction. (b) Enhancement factors calculated from the signals versus $20 \mathrm{ng}$ of mouse IgG. Error bars indicate the standard deviation of 3 measurements.

intensity with the multilayered substrate achieved an enhancement of approximately 58 -fold compared with the unmodified substrate. The enhancement factors obtained with HMDS- and Ag-modified substrates were both less than 5-fold, which indicates 
that both layers of HMDS PPF and Ag are necessary for large enhancement. Note that fluorescence intensities from each spot were calculated by subtracting the background signals of each substrate. Therefore, despite the relatively high background fluorescence, the quantitative analysis of the enhancement factors revealed the apparent effect of the multilayered structure.

\subsection{Immunoassay on protein array prepared with multilayered substrate}

We further performed an immunoassay using a protein array prepared by immobilizing mouse-derived anti-CRP antibody, rabbit-derived anti-CRP antibody, mouse IgG, and rabbit IgG on the multilayered substrate. Cy3-labeled anti-mouse IgG antibody was added to the array to allow interaction with the proteins. Two-dimensional fluorescence images showed that fluorescence signals from mouse-derived anti-CRP antibody as well as those from mouse IgG were highly enhanced on the multilayered substrate compared with those on the unmodified one (Fig. 6). The results indicate that the multilayered substrate can enhance the fluorescence signals derived only from specific immunoreaction among multiple proteins. The enhancement factors of the fluorescence were calculated by subtracting the signals from background intensities of each substrate. The fluorescence intensities from spots of $10 \mathrm{ng}$ protein immobilized on the multilayered substrate achieved an enhancement of approximately 60 -fold for mousederived anti-CRP antibody and 81-fold for mouse IgG compared with those obtained

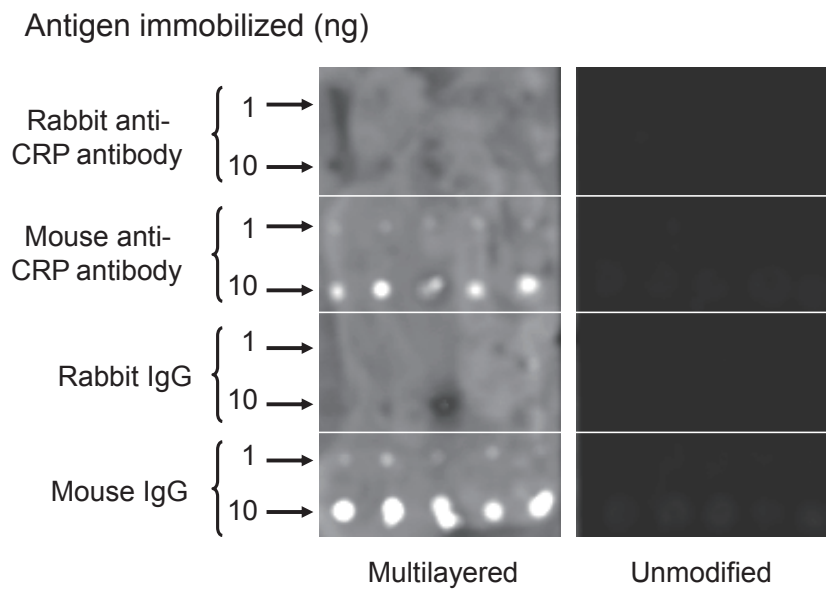

Substrate

Fig. 6. Immunoassay on a protein array prepared with a multilayered substrate. Rabbitderived anti-CRP antibody, mouse-derived anti-CRP anitibody, mouse IgG, and rabbit IgG were immobilized on the surfaces of unmodified substrate and on a modified one with a 61 -nm-thick HMDS PPF. Antigen-antibody interaction was evaluated using Cy3-labeled anti-mouse IgG antibody, and two-dimensional fluorescence images were collected. 
with the unmodified substrate (data not shown). Moreover, while fluorescence from spots of even $10 \mathrm{ng}$ target protein were difficult to observe on the control substrate, as little as $1 \mathrm{ng}$ of two target proteins could be detected on the multilayered substrate. This indicates that the multilayered substrate contributed at least more than 10-fold sensitivity to the immunoassay.

\section{Conclusions}

PPF was used as the optical interference layer on multilayered substrates for fluorescence-based sensitive immunoassay. A glass slide modified with a Ag layer and HMDS PPF was shown to enhance fluorescence intensity from immobilized Cy3labeled antibody. The maximum fluorescence intensity was obtained when the PPF thickness was $63 \mathrm{~nm}$, producing a 30-fold enhancement of fluorescence compared with that obtained from the unmodified substrate. Immunoassay on a protein array prepared with the multilayered substrate also enhanced fluorescence signals derived from specific antigen-antibody interactions. These results demonstrate that HMDS PPF can serve as the optical interference layer on multilayered substrates. As the plasma polymerization technique has already been used for immobilization of biomolecules, it will be further applied to multifunctional sensor devices for highly sensitive detection.

\section{Acknowledgements}

This work was supported by JSPS KAKENHI Grant Number 25410151.

\section{References}

1 K. Kuwabara, M. Ogino, T. Ando and A. Miyauchi: Appl. Phys. Lett. 93 (2008) 33904.

2 K. E. Sapsford, J. B. Blanco-Canosa, P. E. Dawson and I. L. Medintz: Bioconjugate Chem. 21 (2010) 393.

3 D. Darvill, A. Centeno and F. Xie: Phys. Chem. Chem. Phys. 15 (2013) 15709.

4 J. N. Anker, W. P. Hall, O. Lyandres, N. C. Shah, J. Zhao and R. P. V. Duyne: Nat. Mater. 7 (2008) 442.

5 C. R. Sabanayagam and J. R. Lakowicz: Nucleic Acids Res. 35 (2007) e13.

6 Y. Fu and J. R. Lakowicz: Anal. Chem. 78 (2006) 6238.

7 H. Li, M. Wang, W. Qiang, H. Hu, W. Li and D. Xu: Analyst 139 (2014)1653.

8 T. Yamaguchi, T. Kaya and H. Takei: Anal. Biochem. 364 (2007) 171.

9 H. M. Hiep, H. Yoshikawa, M. Saito and E. Tamiya: ACS Nano 3 (2009) 446.

10 H. Szmacinski, K. Ray and J. R. Lakowicz: Anal. Biochem. 385 (2009) 358.

11 W. R. Holland and D. G. Hall: Opt. Lett. 10 (1985) 414.

12 K. G. Sullivan, O. King, C. Sigg and D. G. Hall: Appl. Opt. 33 (1994) 2447.

13 E. Le Moal, E. Fort, S. Lévêsque-Fort, F. P. Cordelières, M.-P. Fontaine-Aupart and C. Ricolleau: Biophys. J. 92 (2007) 2150.

14 T. Akimoto, M. Yasuda and I. Karube: Appl. Opt. 47 (2008) 3789.

15 T. Akimoto and M. Yasuda: Appl. Opt. 49 (2010) 80.

16 H. Etoh, M. Yasuda and T. Akimoto: Anal. Sci. 27 (2011) 1179.

17 M. Yasuda and T. Akimoto: Anal. Sci. 28 (2012) 947. 
18 H. Choumane, N. Ha, C. Nelep, A. Chardon, G. O. Reymond, C. Goutel, G. Cerovic, F. Vallet, C. Weisbuch and H. Benisty: Appl. Phys. Lett. 87 (2005) 31102.

19 S. Kurosawa, N. Kamo, D. Matsui and Y. Kobatake: Anal. Chem. 62 (1990) 353.

20 A. Hiratsuka and I. Karube: Electroanalysis 12 (2000) 695.

21 H. Muguruma: Plasma Processes Polym. 7 (2010) 151.

22 R. Nakamura, H. Muguruma, K. Ikebukuro, S. Sasaki, R. Nagata, I. Karube and H. Pedersen: Anal. Chem. 69 (1997) 4649.

23 K. Kojima, A. Hiratsuka, H. Suzuki, K. Yano, K. Ikebukuro and I. Karube: Anal. Chem. 75 (2003) 1116.

24 H. Miyachi, K. Ikebukuro, K. Yano, H. Aburatani and I. Karube: Biosens. Bioelectron. 20 (2004) 184.

25 H. Kinoshita, S. Ishiwara, Y. Tsuji, M. Dejima, K. Yano, I. Takase and I. Karube: Anal. Chim. Acta 561 (2006) 25.

26 H. Muguruma, A. Hiratsuka and I. Karube: Anal. Chem. 72 (2000) 2671.

27 H. Muguruma, T. Hoshino and Y. Matsui: ACS Appl. Mater. Interfaces 3 (2011) 2445.

28 T. Hoshino, T. Inoue and H. Muguruma: IEICE Trans. Electron. E96-C (2013) 1536.

29 S.-W. Tsai, M. Loughran, A. Hiratsuka, K. Yano and I. Karube: Analyst 128 (2003) 237.

30 H. Muguruma and H. Takahashi: Surf. Coat. Technol. 205 (2010) 2490.

31 H. Miyachi, A. Hiratsuka, K. Ikebukuro, K. Yano, H. Muguruma and I. Karube: Biotechnol. Bioeng. 69 (2000) 323.

32 S. H. Lee and D. C. Lee: Thin Solid Films 352 (1998) 83. 